SISSA-46/95/EP

May 1995

\title{
SUPERSYMMETRIC RADIATIVE CORRECTIONS TO NEUTRINO INDICES OF REFRACTION
}

\author{
Esteban Roulet \\ SISSA, Trieste, 34100, Italy
}

\begin{abstract}
We compute the one-loop effects on the neutrino propagation through matter induced by virtual supersymmetric particles. We show that, in the minimal version of the supersymmetric standard model, a non-degeneracy between sleptons of the second and third generations can have sizeable effects on the $\nu_{\mu}-\nu_{\tau}$ oscillations in matter. In particular, we discuss how this could affect the detection of the energetic neutrino fluxes arising from annihilation of supersymmetric dark matter in the center of the sun.
\end{abstract}


The fact that $W$-boson exchange with electrons in ordinary matter affects the $\nu_{e}$ index of refraction but not those of the other neutrino flavours is the basis of the Mikheyev-SmirnovWolfenstein (MSW) effect [1], which provides the nicest explanation to the solar neutrino problem.

In the Standard Model (SM), at small momentum transfer muon and tau neutrinos interact indistinguishably with ordinary matter at the tree level. However, a difference among the $\nu_{\mu}$ and $\nu_{\tau}$ indices of refraction in matter appears at one-loop [2], although it is suppressed by $O\left(\frac{\alpha}{\pi \sin ^{2} \theta_{W}} \frac{m_{\tau}^{2}}{M_{W}^{2}}\right)$ with respect to the size of the charged current effects affecting the $\nu_{e}$ propagation, having then probably no observable implications.

In this paper we compute the one-loop contributions to the neutrino refraction indices in the best motivated extension of the SM, i.e. the minimal supersymmetric version of it (MSSM). We show that there are potentially much larger radiative effects than in the SM itself and we discuss the possible physical relevance they could have.

The interactions of neutrinos with matter are described by the matrix element

$$
M\left(\nu_{\ell} f \rightarrow \nu_{\ell} f\right)=-i \frac{G_{F}}{\sqrt{2}} \bar{\nu}_{\ell} \gamma^{\rho}\left(1-\gamma_{5}\right) \nu_{\ell} \bar{f} \gamma_{\rho}\left(C_{\nu_{\ell} f}^{V}+C_{\nu_{\ell} f}^{A} \gamma_{5}\right) f
$$

For neutrinos propagating through an unpolarized medium at rest, the temporal component of the fermionic vector current leads to a non-vanishing neutrino forward-scattering amplitude and, hence, to a neutrino refraction index $n_{\nu}$ given by [3]

$$
p_{\nu}\left(n_{\nu_{\ell}}-1\right)=-\sqrt{2} G_{F} \sum_{f=u, d, e} C_{\nu_{\ell} f}^{V} N_{f} .
$$

$N_{f}$ is the number density of fermion $f$ and, at the tree-level,

$$
C_{\nu_{\ell} f}^{V}=T_{3}\left(f_{L}\right)-2 Q_{f} s_{W}^{2}+\delta_{\ell f}
$$

with $s_{W}^{2} \equiv \sin ^{2} \theta_{W}, T_{3}\left(f_{L}\right)$ the third component of isospin of $f_{L}$ and $Q_{f}$ its charge.

The indices of refraction affect the neutrino flavour evolution during propagation, which is described by (for reviews see [4])

$$
i \frac{d}{d t}\left(\begin{array}{c}
\nu_{e} \\
\nu_{\mu} \\
\nu_{\tau}
\end{array}\right)=\left[\frac{1}{2 p_{\nu}} V\left(\begin{array}{ccc}
\Delta m_{12}^{2} & 0 & 0 \\
0 & 0 & 0 \\
0 & 0 & \Delta m_{32}^{2}
\end{array}\right) V^{\dagger}-p_{\nu}\left(\begin{array}{ccc}
\Delta n_{e \mu} & 0 & 0 \\
0 & 0 & 0 \\
0 & 0 & \Delta n_{\tau \mu}
\end{array}\right)\right]\left(\begin{array}{c}
\nu_{e} \\
\nu_{\mu} \\
\nu_{\tau}
\end{array}\right)
$$

where $V$ is the unitary matrix relating the neutrino flavour $\left(\nu_{\alpha}\right.$, with $\left.\alpha=e, \mu, \tau\right)$ and mass $\left(\nu_{i}\right.$, with $\left.i=1,2,3\right)$ eigenstates, i.e. $\nu_{\alpha}=V_{\alpha i} \nu_{i}$. Also $\Delta m_{i j}^{2} \equiv m_{\nu_{i}}^{2}-m_{\nu_{j}}^{2}$ and $\Delta n_{\alpha \beta} \equiv n_{\nu_{\alpha}}-n_{\nu_{\beta}}$. (We note that, although the indices of refraction will be computed in the MSSM, some departure from it should be responsible for the neutrino masses and mixings themselves). 
Since matter effects in oscillations involving $\nu_{e}$ will be largely dominated by the charged current (CC) term $\delta_{\ell f}$ in eq. (3), the radiative corrections to the tree-level result $\Delta n_{e \mu}=$ $-\sqrt{2} G_{F} N_{e} / p_{\nu}$ turn out to be negligible. We will then concentrate on the computation of $\Delta n_{\tau \mu}$, which does not involve the CC piece and vanishes at the tree-level.

It is useful to parametrise the effects of the radiative corrections, for $f \neq \ell$, as

$$
C_{\nu_{\ell} f}^{V}=\rho^{\nu_{\ell} f} T_{3}\left(f_{L}\right)-2 Q_{f} \lambda^{\nu_{\ell} f} s_{W}^{2}
$$

The splitting of the radiative effects between $\rho$ and $\lambda$ is somewhat arbitrary, and it is convenient to include in $\rho$ the $f$-dependent box diagram contributions. This has the advantage that, for a neutral medium, the corrections in $\lambda$ (which include also contributions from the neutrino charge radius [5]) turn out to be $f$-independent (see ref. [2]) and do not contribute to $\Delta n_{\tau \mu}$, due to the fact that

$$
\sum_{f=u, d, e} N_{f} Q_{f}=0
$$

One has then

$$
p_{\nu} \Delta n_{\tau \mu}=-\sqrt{2} G_{F} \sum_{f} N_{f} T_{3}\left(f_{L}\right) \Delta \rho^{f}
$$

with $\Delta \rho^{f} \equiv \rho^{\nu_{\tau} f}-\rho^{\nu_{\mu} f}$.

In the SM, $\Delta \rho^{f}$ gets contributions from the one-loop corrections to the $\bar{\nu} \nu Z$ vertex and $W$-boson box diagrams [2], leading to

$$
\begin{gathered}
\Delta \rho_{S M}^{e}=\Delta \rho_{S M}^{d}=\frac{\alpha_{W}}{8 \pi}\left[\frac{x(2+x)}{1-x}+\frac{3 x(2-x)}{(1-x)^{2}} \ln x\right] \\
\Delta \rho_{S M}^{u}=\frac{-\alpha_{W}}{8 \pi}\left[\frac{x(4-x)}{1-x}+\frac{3 x^{2}}{(1-x)^{2}} \ln x\right]
\end{gathered}
$$

with $x \equiv m_{\tau}^{2} / M_{W}^{2}$ and $\alpha_{W} \equiv \alpha / s_{W}^{2}$. These corrections are small due to the one-loop factor $\alpha_{W} / 4 \pi$, but are also quite suppressed by the smallness of the factor $m_{\tau}^{2} / M_{W}^{2} \sim 4 \times 10^{-4}$.

The computation of the supersymmetric contribution to $\Delta \rho$ requires the evaluation of the Feynman diagrams depicted in fig. 1, leading to the results that are summarized in the Appendix. The important point is that, besides the one-loop factor $\alpha_{W} / 4 \pi$, what fixes now the size of $\Delta \rho$ is mainly the splitting among the sleptons of the second and third generations.

At this point, it is useful to recall that a usual simplifying assumption made in phenomenological applications is to consider all sfermions to be exactly degenerate at the GUT scale, and obtain their low energy splittings from the renormalization group evolution of the soft parameters and from terms arising after the electroweak symmetry breaking. In this way, although squarks get significantly splitted from sleptons, the splittings among the 
masses of different slepton generations are only due to the small $\tau$-Yukawa coupling. This usually implies that $m_{\tilde{\tau}}^{2}-m_{\tilde{\mu}}^{2}$ is $O\left(m_{\tau}^{2}\right)$, and hence the radiative effects on the $\nu_{\mu, \tau}$ indices of refraction are in this case not larger than the SM ones. An exception to this, still assuming a universal soft scalar mass $m$, is when there is a large $\tilde{\tau}_{L}-\tilde{\tau}_{R}$ mixing. This happens for large values of the Higgs mixing parameter $\mu$ and large $\operatorname{tg} \beta$, or for large values of the parameter $A$ of the trilinear soft terms, in which case the splitting can be $O\left[m_{\tau}(A m+\mu \operatorname{tg} \beta)\right]$ (see ref. [6]).

From a more general perspective, the universality assumptions (which give the easiest way to get rid of FCNC phenomena) are not really a necessity, and actually non-universal soft terms usually arise in string theories [7] and can also be generated in GUTs [8]. Universal sfermion masses may not even be desirable in some respects, and it has been argued that non-universalities may prove useful in reconciling different phenomenological constraints in supersymmetric GUTs [9, 8]. Also, it has recently been suggested that sfermion masses may dynamically align along the directions, in flavour space, of the fermion masses, suppressing FCNC but allowing large mass splittings [10].

If one considers the general case in which a sizeable splitting is allowed among $\mu$ and $\tau$ sleptons $\mathrm{f}^{\mathrm{m}}$, the SUSY contribution to $\Delta n_{\tau \mu}$ could then be larger than the SM one.

We will present an illustrative situation in which the effect here described is important and then comment on how the results are modified when one changes the starting assumptions. For simplicity we assume no $\tilde{f}_{L}-\tilde{f}_{R}$ mixings, neglect intergenerational mixings of sleptons as well as splittings due to $D$-terms among charged and neutral sleptons or among $\tilde{\ell}_{L}$ and $\tilde{\ell}_{R}$, which are anyhow inessential to the conclusions reached. We take first generation sleptons degenerate with the second generation ones, and only allow the third generation sleptons to have a different mass. We take a light but experimentally allowed value for the second generation slepton masses, $m_{\tilde{\mu}}=m_{\tilde{\nu}_{\mu}}=\operatorname{Max}\left[60 \mathrm{GeV}, m_{\chi}+20 \mathrm{GeV}\right]$, where $m_{\chi}$ is the lightest neutralino mass (which we assume to be the lightest supersymmetric particle (LSP)), and take second and third generation sleptons to be splitted by an amount $m_{\tilde{\tau}}-m_{\tilde{\mu}}=60 \mathrm{GeV}$. We also assume that squarks are much heavier than sleptons (as usually results from the effects of gluino masses in the renormalization group evolution of scalar masses). This last implies that box diagrams only contribute sizeably to interactions with electrons.

In fig. 2 we plot the ratio of the SUSY and SM values of $\Delta n_{\tau \mu}$ for an isoscalar medium $\left(Y_{n} \equiv N_{n} / N_{p}=1\right)$, as a function of the supersymmetric parameter space that determines the chargino and neutralino masses and couplings ( $\mathrm{SU}(2)$ gaugino mass $M$ and Higgs mixing parameter $\mu$ ). We present results for values of the ratio of Higgs VEVs $\operatorname{tg} \beta \equiv v_{2} / v_{1}=2$

\footnotetext{
${ }^{1}$ This splitting is not directly related to very suppressed rare processes such as $\mu \rightarrow e \gamma, \mu \rightarrow 3 e, \tau \rightarrow \mu \gamma$, etc. [11], but could give rise to small universality violations [12].

${ }^{2}$ for definiteness we assumed common gaugino masses at the GUT scale to obtain the neutralino spectra
} 
(fig. 2.a) and $\operatorname{tg} \beta=40$ (fig. 2.b), showing that the dependence on it is only mild. The dark regions for small values of $\mu$ and $M$ are excluded by the LEP constraint $m_{\chi^{+}}>45 \mathrm{GeV}$, that is the main bound from accelerators.

It is apparent from fig. 2 that, for the slepton mass splittings considered, $\Delta n_{\tau \mu}$ may be an order of magnitude larger than in the SM. The SUSY contribution turns out to be dominated by the chargino boxes and penguins involving $\tilde{\ell}_{L}$ exchange. The neutralino boxes are generally small, while neutralino penguins, not shown in fig. 1, give no contribution (similarly to what happens for instance in $b \rightarrow s \ell^{+} \ell^{-}$decays 13]). Thus, the relevant splitting is the one among charged sleptons rather than among sneutrinos. The effect is especially large in a region of parameter space where the chargino masses are below 80$100 \mathrm{GeV}$, i.e. testable at LEPII, and becomes less important for large values of $|\mu|$ and $M$, i.e. for heavier charginos. Slepton splittings smaller than the one adopted would lead to proportionally smaller effects, while larger splittings can increase the effect by up to a factor of two. The neglected box diagrams involving squark exchange may also increase the SUSY contribution. Also note that, since penguin contributions to $\Delta \rho$ are $f$-independent, they lead (see eq. (7)) to a contribution to $\Delta n_{\tau \mu}$ proportional to $N_{e}\left(2-Y_{n}\right)$ (in the sun, $Y_{n}$ varies from $\sim 0.16$ in the surface to 0.5 in the center).

Clearly the sign of $\Delta n_{\tau \mu}$, and hence whether resonant matter effects take place among neutrinos or antineutrinos], depends, for significant slepton splittings, on whether $\tilde{\tau}$ are heavier or lighter than $\tilde{\mu}\left(m_{\tilde{\tau}}\right.$ smaller than $m_{\tilde{\mu}}$ leads to a resonance crossing among neutrinos if $\left.\Delta m_{32}^{2}>0\right)$.

Let us also note that the penguin and box diagrams involving $\tilde{\ell}_{R}$ exchange are proportional to the square of the lepton Yukawa coupling, so that their effect on $n_{\nu_{\mu}}$ is much smaller than that on $n_{\nu_{\tau}}$. This fact has the interesting effect of making their contribution to $\Delta n_{\tau \mu}$ to depend just on $m_{\tilde{\tau}}$, rather than on a slepton mass splitting. However, even for $\operatorname{tg} \beta=40$ and $m_{\tilde{\tau}_{R}}=60 \mathrm{GeV}$, they give a contribution not larger than the SM one. Similar conclusions hold for the penguins involving $H^{+}$exchange (keeping in mind that in the MSSM $m_{H^{+}}>M_{W}$ ).

We finally mention that other extensions of the SM may also sizeably affect $\Delta n_{\tau \mu}$. In particular, supersymmetric $R$-parity violating interactions can modify the neutrino indices of refraction already at the tree-level [14], although those models would be less interesting as regards the application discussed below. Another simple example would be the presence of a new $Z^{\prime}$ gauge boson with non-universal couplings to leptons [15].

We turn now to consider the possible physical relevance of these radiative effects for $\nu_{\mu}-$ $\nu_{\tau}$ matter oscillations. When discussing applications, we will neglect the $\nu_{\mu, \tau}$ mixing with

\footnotetext{
${ }^{3}$ for antineutrinos, the sign of the matrix element is reversed, so that $n_{\bar{\nu}_{\tau}}-n_{\bar{\nu}_{\mu}}=-\left(n_{\nu_{\tau}}-n_{\nu_{\mu}}\right)$
} 
$\nu_{e}$ to be left with just a two flavour situation. The generalization to three flavour neutrino mixing should pose no problems.

A first difficulty to observe any conversion among $\nu_{\mu}$ and $\nu_{\tau}$ is that for low energies, $E_{\nu}<$ $0.1 \mathrm{GeV}$, these neutrinos are only detected by means of their neutral current interactions and are hence essentially indistinguishable. Furthermore, only e-type neutrinos are produced in the sun (except for possible ordinary MSW conversions inside the sun) and equal amounts of $\nu_{\mu}$ and $\nu_{\tau}$ are produced in supernovae, so that oscillations among them do not give actually any overall result. These problems are not present in long-baseline $\nu_{\mu}$ oscillation experiments on earth (either with $\nu_{\mu}$ from accelerators or using atmospheric neutrinos), but it is easy to convince oneself that the resonance oscillation length in terrestrial matter (inversely proportional to $\Delta n_{\tau \mu}$ ) is typically much larger than the earth diameter, and hence oscillation effects are negligible.

The situation that we want to describe, in which the matter effects here analysed are relevant, is actually directly related to the supersymmetric framework under consideration. It is well known that a nice feature of supersymmetry, once $R$-parity conservation is adopted to avoid $B$ and $L$ violation, is that the LSP, usually a neutralino, is stable and naturally becomes a good dark matter (DM) candidate. There are two main strategies that are being pursued at present to experimentally search for SUSY DM [16]. The first is the direct search of the energy deposited by halo neutralinos interacting with target nuclei, and the second one is the search of energetic neutrinos produced in the annihilation of DM trapped in the interior of the sun or the earth [17] In particular, upward-going muons produced in the rock (or ice) just below underground detectors by energetic $\nu_{\mu}$ and $\bar{\nu}_{\mu}$ (with $E_{\nu}>$ few $\mathrm{GeV}$ ) may allow to probe significant regions of the supersymmetric parameter space in new installations such as Superkamiokande or Amanda. As was shown in ref. [18, the usual MSW effect between $\nu_{e}$ and $\nu_{\mu, \tau}$ can affect the detection rate predictions.

To show the possible effects of energetic $\nu_{\mu}-\nu_{\tau}$ matter enhanced oscillations in the solar interior, we plot in fig. 3 the $\nu_{\mu}$ neutrino survival probability in the $\sin ^{2} 2 \theta_{\mu \tau}$ vs. $\Delta m_{32}^{2}$ plane, assuming that $\epsilon \equiv \Delta n_{\tau \mu} / \Delta n_{\mu e}=10^{-3}$ (in the SM, $\epsilon \simeq-5 \times 10^{-5}$ ). The contours correspond to $P\left(\nu_{\mu} \rightarrow \nu_{\mu}\right)=0.8$ (continuous lines) and 0.45 (dashed lines) for two neutrino energiesf $E_{\nu}=10$ and $40 \mathrm{GeV}$. It is clear that, for significant ranges of $\Delta m^{2}$ and $\sin ^{2} 2 \theta$, the oscillations of high energy neutrinos are sizeably affected by matter effects. For decreasing values of $|\epsilon|$, the MSW type resonant effects take place for smaller $\Delta m^{2}$ values. The adiabatic condition in the resonance transition becomes harder to achieve, making the regions of significant

\footnotetext{
${ }^{4}$ halo $\chi$ annihilations may also provide some signals.

${ }^{5}$ for energies above $100 \mathrm{GeV}, \nu$ absorption in the sun starts to be relevant, making the oscillation formalism to be no longer valid. Our computation of the box diagrams, which neglected external momenta, actually contained terms proportional to $p_{\nu} \cdot p_{f}$, which would modify the results only for $E_{\nu}>$ few $\mathrm{TeV}$.
} 
transition to shrink towards large mixings, and the effect eventually becomes very small for $|\epsilon|<10^{-4}$.

These $\nu_{\mu}-\nu_{\tau}$ oscillations may have important implications for the detection of neutralino annihilation signals. This is because the $\nu_{\mu}$ and $\nu_{\tau}$ fluxes from neutralino annihilation are generally quite different, so that oscillations among them modify the expected $\nu_{\mu}$ signal at underground detectors. The difference among $\nu_{\mu}$ and $\nu_{\tau}$ fluxes has its origin in the fact that the non-relativistic neutralino annihilation cross section into fermion pairs $f \bar{f}$ is proportional to $m_{f}^{2}$, either due to a $p$-wave suppression (as for annihilations mediated by $Z$ or sfermion exchange) or due to a Yukawa suppression (as in the case of Higgs boson mediated annihilations). Hence, neutralinos do not directly annihilate into neutrino pairs. Different neutrino fluxes result then from $b, c$ and $\tau$ decays [19] (for $m_{\chi}<M_{W}$, since otherwise other channels involving gauge bosons in the final state are also allowed and can produce prompt secondary neutrinos of different flavours in similar amounts). Furthermore, light mesons and muons produced in the $\chi$ annihilation are stopped by the solar medium before they decay, yielding no secondary fluxes of energetic neutrinos.

Rather than scanning all the supersymmetric parameter space, we will consider as an illustrative example the simple but still quite general case in which the lightest neutralino is mainly gaugino, i.e. $|\mu|>M$, with $m_{\chi}<M_{W}$ (in this region the effect here discussed is potentially large and also the neutralino cosmological relic density is usually significant). If squarks are heavier than sleptons, as we are assuming, and sleptons are not too heavy, the main non-relativistic neutralino annihilation channel is by $t$-channel $\tilde{\tau}$ exchange, producing a $\tau \bar{\tau}$ pair. The $\nu_{\tau}$ and $\nu_{\mu}$ fluxes from the subsequent $\tau$ decays will then clearly be quite different. (In the general case of an arbitrary neutralino composition and squark masses, the fluxes are still different but one needs to include the extra annihilation channels and the model dependent branching ratios entering in the $\nu_{\mu, \tau}$ yields).

In fig. 4 we show the $\nu_{\tau}$ and $\nu_{\mu}$ differential neutrino yields (with thin dashed and solid lines respectively) produced by the annihilation of neutralinos into a $\tau$ pair (the main annihilation channel in our example). What is actually plotted is $z^{2} d N / d z$ (where $z \equiv E_{\nu} / m_{\chi}$ ), which is the relevant quantity for underground $\nu_{\mu}$ detection' because both the CC $\nu_{\mu}$ cross section and the muon range in the rock (or ice) are proportional to the neutrino energy.

With thick lines we show how the fluxes get modified after traversing the solar interior, assuming $m_{\chi}=50 \mathrm{GeV}$ and taking $\left(\epsilon, \Delta m^{2}, \sin ^{2} 2 \theta\right)$ to be $\left(10^{-3}, 6 \times 10^{-4} \mathrm{eV}^{2}, 0.1\right)$ in fig. 4.a, $\left(10^{-3}, 3 \times 10^{-4} \mathrm{eV}^{2}, 0.1\right)$ in fig. $4 . b$ and $\left(10^{-3}, 10^{-3} \mathrm{eV}^{2}, 0.6\right)$ in fig. 4.c. The neutrino masses and mixings assumed in the case of fig. 4.c lie in the region, also shown in fig. 3, of interest to explain the atmospheric neutrino anomaly. One should note, however, that for large

\footnotetext{
${ }^{6}$ the muon flux arising from $\nu_{\tau}$ interactions is quite suppressed
} 
mixings the effects of vacuum oscillations are significant and would affect both neutrinos and antineutrinos, while matter induced oscillations affect either one or the other.

From the results, one can see that the detection rates may be sizeably modified by the matter enhanced oscillations here described, providing an interesting physical manifestation of the radiative supersymmetric effects studied. The uncertainties involved in the theoretical predictions of the DM annihilation signal (unknown neutralino mass and composition, uncertainties in the local halo density and DM velocity distribution, etc.) will however complicate the interpretation of any positive detection, so that these effects should actually be considered as providing a further spread in the theoretical predictions until these parameters become more constrained by accelerators and direct DM searches. The shape of the neutrino spectrum gives probably a clearer signature of the matter oscillations, and may provide a useful handle to identify them.

I would like to thank Stefano Bertolini for very useful discussions.

\section{Appendix}

We summarize here the supersymmetric contributions to $\Delta n_{\tau \mu}$ arising from the diagrams in fig. 1.

A few simplifying assumptions will be adopted. Motivated by the smallness of FCNC phenomena, we will assume that sfermion masses align, in flavour space, in the directions of the fermion masses. This makes the neutralino-fermion-sfermion vertices diagonal in generation space. We will also ignore $\tilde{f}_{L}-\tilde{f}_{R}$ mixings, so that the appropriate mass eigenstates are $\tilde{f}_{i L}$ and $\tilde{f}_{i R}$. These assumptions simplify the calculations but are not essential to the conclusions reached.

Following the notation of ref. [6], we denote by $Z_{i j}^{\prime}$ the $4 \times 4$ matrix diagonalizing the neutralino states in the basis $\left(\tilde{\gamma}, \tilde{Z}, \tilde{H}_{1}, \tilde{H}_{2}\right)$, and $U$ and $V$ are the $2 \times 2$ matrices required for the diagonalization of the chargino mass matrix. In the radiative corrections involving the neutralinos $\chi_{i}^{0}$, only the gaugino components will contribute sizeably since the higgsino couplings are very small for interactions with ordinary matter. The Feynman rule for the $\bar{f} \chi_{j}^{0} \tilde{f}_{L}$ vertex can be then parametrised as $-i g \sqrt{2} G_{f L}^{j *} P_{R}$ while that involving $\tilde{f}_{R}$ by $i g \sqrt{2} G_{f R}^{j *} P_{L}$. The couplings $G^{j}$ to the gaugino components are given by

$$
\begin{gathered}
G_{f L}^{j}=Q_{f} s_{W} Z_{j 1}^{\prime *}+\frac{c_{L}^{f}}{c_{W}} Z_{j 2}^{* *} \\
G_{f R}^{j}=\operatorname{sign}\left(m_{\chi_{j}^{0}}\right)\left[Q_{f} s_{W} Z_{j 1}^{\prime}+\frac{c_{R}^{f}}{c_{W}} Z_{j 2}^{\prime}\right]
\end{gathered}
$$


with $c_{L(R)}^{f}=T_{3}\left(f_{L(R)}\right)-Q_{f} s_{W}^{2}$.

Regarding the chargino couplings, we will ignore Cabibbo-type intergenerational mixings (for leptons] and, in the box contributions also for quarks), since the flavour-conserving processes under study are not GIM suppressed. We then only include the chargino mixing matrices in the vertices (e.g. the $\bar{\nu}_{\ell} \chi_{j}^{+} \tilde{\ell}_{L}$ vertex is $-i g U_{j 1} P_{R}$, the $\bar{\nu}_{\ell} \chi_{j}^{+} \tilde{\ell}_{R}$ vertex is $i g H_{j}^{\ell} P_{R}$, with $H_{j}^{\ell} \equiv m_{\ell} U_{j 2} / \sqrt{2} M_{W} c \beta$, see ref. [6]).

The penguin and box contributions to $\Delta \rho^{f}$ can be written as

$$
\Delta \rho^{f}=\Delta \rho_{p}+\frac{\Delta \rho_{b o x}^{f}}{T_{3}\left(f_{L}\right)} .
$$

The ( $f$-independent) contributions to the penguins involving charginos and $\tilde{\ell}_{L}$ exchange, arise from the self-energy diagrams in fig. 1.a, $\Delta \rho_{p}^{L}(\Sigma)$, and from the diagrams where the $Z$ couples to the slepton-line $\Delta \rho_{p}^{L}(\tilde{\ell})$ (fig. 1.b), or to the chargino-line, $\Delta \rho_{p}^{L}\left(\chi^{+}\right)$(fig. 1.c). Direct computation leads to the results:

$$
\begin{aligned}
\Delta \rho_{p}^{L}(\Sigma)= & \frac{\alpha_{W}}{8 \pi} \sum_{j=1}^{2}\left|U_{j 1}\right|^{2}\left\{G_{0}\left(X_{\chi_{j}^{+} \tilde{\tau}_{L}}, 1\right)+\ln \frac{m_{\tilde{\tau}_{L}}^{2}}{\mu^{2}}-\left(\tilde{\tau}_{L} \rightarrow \tilde{\mu}_{L}\right)\right\} \\
\Delta \rho_{p}^{L}(\tilde{\ell})=- & \frac{\alpha_{W}}{4 \pi} c_{L}^{\ell} \sum_{j=1}^{2}\left|U_{j 1}\right|^{2}\left\{G_{0}\left(X_{\chi_{j}^{+} \tilde{\tau}_{L}}, 1\right)+\ln \frac{m_{\tilde{\tau}_{L}}^{2}}{\mu^{2}}-\left(\tilde{\tau}_{L} \rightarrow \tilde{\mu}_{L}\right)\right\} \\
\Delta \rho_{p}^{L}\left(\chi^{+}\right)= & -\frac{\alpha_{W}}{4 \pi} \sum_{i, j=1}^{2} U_{i 1} U_{j 1}^{*}\left\{2 \mathcal{O}^{\prime L}{ }_{i j}^{L} \sqrt{X_{\chi_{i}^{+} \tilde{\tau}_{L}} X_{\chi_{j}^{+} \tilde{\tau}_{L}}} F_{0}\left(X_{\chi_{i}^{+} \tilde{\tau}_{L}}, X_{\chi_{j}^{+} \tilde{\tau}_{L}}\right)-\right. \\
& \left.\mathcal{O}_{i j}^{\prime R}\left[G_{0}\left(X_{\chi_{i}^{+} \tilde{\tau}_{L}}, X_{\chi_{j}^{+} \tilde{\tau}_{L}}\right)+\ln \frac{m_{\tilde{\tau}_{L}}^{2}}{\mu^{2}}\right]-\left(\tilde{\tau}_{L} \rightarrow \tilde{\mu}_{L}\right)\right\},
\end{aligned}
$$

where $X_{a b} \equiv\left(m_{a} / m_{b}\right)^{2}$ and $\mu$ is an arbitrary mass scale. The couplings $\mathcal{O}^{\prime L}{ }_{i j}^{L}=-V_{i 1} V_{j 1}^{*}-$ $\frac{1}{2} V_{i 2} V_{j 2}^{*}+\delta_{i j} s_{W}^{2}$ and $\mathcal{O}_{i j}^{\prime R}=-U_{i 1} U_{j 1}^{*}-\frac{1}{2} U_{i 2} U_{j 2}^{*}+\delta_{i j} s_{W}^{2}$ describe the $\chi_{i}^{+} \chi_{j}^{+} Z$ vertex, that reads $i \frac{g}{c_{W}} \gamma^{\mu}\left[\mathcal{O}_{i j}^{\prime L} P_{L}+\mathcal{O}_{i j}^{\prime R} P_{R}\right]$. The functions $F_{0}$ and $G_{0}$ are

$$
\begin{gathered}
F_{0}(x, y)=\frac{x \ln x}{(x-y)(x-1)}+(x \leftrightarrow y), \\
G_{0}(x, y)=\left[\frac{x^{2} \ln x}{(x-y)(x-1)}+(x \leftrightarrow y)\right]-\frac{3}{2} .
\end{gathered}
$$

The penguins involving $\tilde{\ell}_{R}$ exchange, that although proportional to $\left(m_{\tau} / M_{W}\right)^{2}$ may in principle be enhanced for large values of $\operatorname{tg} \beta$, give a total contribution (neglecting the $\tilde{\mu}_{R}$

\footnotetext{
${ }^{7}$ this may not be a good approximation for neutrino mixings close to maximal
} 
exchange that is proportional to $\left.m_{\mu}^{2} / M_{W}^{2}\right)$

$$
\begin{aligned}
\Delta \rho_{p}^{R} \simeq & \frac{\alpha_{W}}{4 \pi} \sum_{j=1}^{2}\left\{\left|H_{j}^{\tau}\right|^{2}\left(\frac{1}{2}-s_{W}^{2}\right) G_{0}\left(X_{\chi_{j}^{+} \tilde{\tau}_{R}}, 1\right)+\sum_{i=1}^{2} H_{i}^{\tau} H_{j}^{\tau *}\left[\mathcal{O}_{i j}^{\prime R} \times\right.\right. \\
& {\left.\left.\left[G_{0}\left(X_{\chi_{i}^{+} \tilde{\tau}_{R}}, X_{\chi_{j}^{+} \tilde{\tau}_{R}}\right)+1\right]-2 \mathcal{O}^{\prime L}{ }_{i j}^{L} \sqrt{X_{\chi_{i}^{+} \tilde{\tau}_{R}} X_{\chi_{j}^{+} \tilde{\tau}_{R}}} F_{0}\left(X_{\chi_{i}^{+} \tilde{\tau}_{R}}, X_{\chi_{j}^{+} \tilde{\tau}_{R}}\right)\right]\right\} . }
\end{aligned}
$$

Similarly, the charged Higgs boson contribution in fig. 1.a-c is

$$
\Delta \rho_{p}^{H^{+}} \simeq \frac{\alpha_{W}}{4 \pi} \frac{m_{\tau}^{2}}{M_{W}^{2}} \operatorname{tg}^{2} \beta \frac{y}{2}\left[\frac{1}{1-y}+\frac{\ln y}{(1-y)^{2}}\right],
$$

where $y \equiv\left(m_{\tau} / M_{H^{+}}\right)^{2}$.

The box diagrams (fig. 1.d) involving charginos and $\tilde{\ell}_{L}$ exchange give:

$$
\begin{gathered}
\Delta \rho_{b o x}^{e}\left(\chi^{+}\right)=-\frac{\alpha_{W}}{4 \pi} \frac{M_{W}^{2}}{m_{\tilde{\nu}_{e}}^{2}} \sum_{j, k=1}^{2} V_{j 1}^{*} V_{k 1} U_{j 1}^{*} U_{k 1} \times \\
\\
\left\{\sqrt{X_{\chi_{j}^{+} \tilde{\nu}_{e}} X_{\chi_{k}^{+} \tilde{\nu}_{e}}} F^{\prime}\left(X_{\chi_{j}^{+} \tilde{\nu}_{e}}, X_{\chi_{k}^{+} \tilde{\nu}_{e}}, X_{\tilde{\tau}_{L} \tilde{\nu}_{e}}\right)-\left(\tilde{\tau}_{L} \rightarrow \tilde{\mu}_{L}\right)\right\}, \\
\Delta \rho_{b o x}^{d}\left(\chi^{+}\right)=\Delta \rho_{b o x}^{e}\left(\chi^{+}\right)\left(\tilde{\nu}_{e} \rightarrow \tilde{u}_{L}\right), \\
\Delta \rho_{b o x}^{u}\left(\chi^{+}\right)=\frac{\alpha_{W}}{8 \pi} \frac{M_{W}^{2}}{m_{\tilde{d}_{L}}^{2}} \sum_{j, k=1}^{2} U_{j 1} U_{k 1}^{*} U_{j 1}^{*} U_{k 1}\left\{G^{\prime}\left(X_{\chi_{j}^{+} \tilde{d}_{L}}, X_{\chi_{k}^{+} \tilde{d}_{L}}, X_{\tilde{\tau}_{L} \tilde{d}_{L}}\right)-\left(\tilde{\tau}_{L} \rightarrow \tilde{\mu}_{L}\right)\right\} .
\end{gathered}
$$

The boxes with $\tilde{\ell}_{R}$ exchange are just obtained by replacing in the previous expressions $\tilde{\ell}_{L} \rightarrow \tilde{\ell}_{R}, U_{j 1}^{*} U_{k 1} \rightarrow H_{j}^{\tau} H_{k}^{\tau *}$ and omitting the $\tilde{\mu}_{R}$ exchange contribution.

Finally, box diagrams involving neutralinos (fig. 1.e, $f$ ) interacting with $f=e, u, d$ give:

$$
\begin{aligned}
\Delta \rho_{b o x}^{f}\left(\chi^{0}\right)= & -\frac{\alpha_{W}}{\pi} \sum_{j, k=1}^{4} G_{\nu L}^{k} G_{\nu L}^{* j}\left\{\frac { M _ { W } ^ { 2 } } { m _ { \tilde { f } _ { L } } ^ { 2 } } \left[G_{f L}^{k} G_{f L}^{* j} \sqrt{X_{\chi_{j}^{0} \tilde{f}_{L}} X_{\chi_{k}^{0} \tilde{f}_{L}}} F^{\prime}\left(X_{\tilde{\nu}_{\tau} \tilde{f}_{L}}, X_{\chi_{j}^{0} \tilde{f}_{L}}, X_{\chi_{k}^{0} \tilde{f}_{L}}\right)_{-}\right.\right. \\
& \left.\left.\frac{G_{f L}^{j} G_{f L}^{* k}}{2} G^{\prime}\left(X_{\tilde{\nu}_{\tau} \tilde{f}_{L}}, X_{\chi_{j}^{0} \tilde{f}_{L}}, X_{\chi_{k}^{0} \tilde{f}_{L}}\right)\right]+(j \leftrightarrow k, L \leftrightarrow R)\right\}-\left(\tilde{\nu}_{\tau} \rightarrow \tilde{\nu}_{\mu}\right),
\end{aligned}
$$

where

$$
\begin{gathered}
F^{\prime}(x, y, z)=-\frac{x \ln x}{(x-y)(x-z)(x-1)}-\frac{y \ln y}{(y-x)(y-z)(y-1)}-\frac{z \ln z}{(z-x)(z-y)(z-1)} \\
G^{\prime}(x, y, z)=\frac{x^{2} \ln x}{(x-y)(x-z)(x-1)}+\frac{y^{2} \ln y}{(y-x)(y-z)(y-1)}+\frac{z^{2} \ln z}{(z-x)(z-y)(z-1)} .
\end{gathered}
$$




\section{References}

[1] Wolfenstein L., Phys. Rev. D17 (1978) 2369; Mikheyev S. and Smirnov A. Yu, Sov. J. Nucl. Phys. 42 (1985) 913.

[2] Botella F. J., Lim C. S. and Marciano W. J., Phys. Rev. D35 (1987) 896.

[3] Langacker P., Leveille J. P. and Sheiman J, Phys. Rev. D27 (1983) 1228.

[4] Kuo T. K. and Pantaleone J., Rev. Mod. Phys. 61 (1989) 937; Gelmini G. and Roulet E., preprint CERN-TH.7541/94.

[5] Marciano W. J. and Sirlin A., Phys. Rev. D22 (1980) 2695; Sehgal L. M., Phys. Lett. 162B (1985) 370; Ng K. L., Z. Phys. C55 (1992) 145.

[6] Gunion J. and Haber H. E., Nucl. Phys. B272 (1986) 1; Haber H. and Kane G. L., Phys. Rep. 117 (1985) 75.

[7] Ibañez L. and Lüst D., Nucl. Phys. B382 (1992) 305; Kaplunovsky V. and Louis J., Phys. Lett. B306 (1993) 269.

[8] Dimopoulos S. and Pomarol A., preprint CERN-TH/95-44.

[9] Olechowski M. and Pokorski S., Phys. Lett. B344 (1995) 201.

[10] Dimopoulos S. and Giudice G., preprint CERN-TH/95-90.

[11] Choudury D. et al., Phys. Lett. B342 (1995) 180; Gabbiani F. and Masiero A., Nucl. Phys. B322 (1989) 235.

[12] Chankowski P. H., Hempfling R. and Pokorski S., Phys. Lett. B333 (1994) 403.

[13] Bertolini S. et al., Nucl. Phys. B353 (1991) 591.

[14] Roulet E., Phys. Rev. D44 (1991) R935.

[15] Nardi E., Phys. Rev. D48 (1993) 3277.

[16] Primack J., Sadoulet B. and Seckel D., Ann. Rev. Nucl. Part. Sci. B38 (1988) 751.

[17] Silk J., Olive K. and Srednicki M., Phys. Rev. Lett. 55 (1985) 257; Hagelin J., Ng K. W. and Olive K., Phys. Lett. B180 (1986) 375; Srednicki M., Olive K. and Silk J., Nucl. Phys. B279 (1987) 138; Ellis J., Flores R. and Ritz S., Phys. Lett. B198 (1987) 393; Freese K., Phys. Lett. B167 (1986) 295; Krauss L., Srednicki M. and Wilczeck F., Phys. 
Rev. D33 (1986) 2079; Giudice G. and Roulet E., Nucl. Phys. B316 (1989) 429; Gelmini G., Gondolo P. and Roulet E., Nucl. Phys. B351 (1991) 623; Kamionkowski M., Phys. Rev. D44 (1991) 3021; Halzen F., Stelzer T. and Kamionkowski M., Phys. Rev. D45 (1992) 4439; Bottino A. et al., Astropart. Phys. 3 (1995) 65.

[18] Ellis J., Flores R. and Masood S., Phys. Lett. B294 (1992) 229.

[19] Ritz S. and Seckel D., Nucl. Phys. B304 (1988) 877.

\section{Figure Captions}

Fig. 1: Feynman diagrams describing the supersymmetric contribution to $\nu_{\ell^{-}} f$ forward scattering $(\ell=\mu, \tau)$. The blob in fig. 1.a represents corrections to both $\nu$ external legs.

Fig. 2: Ratio of the supersymmetric and SM values of $\Delta n_{\tau \mu}$ for an isoscalar medium. We take the sleptons of the first two generations to have a common mass of Max $[60 \mathrm{GeV}$, $\left.m_{\chi}+20 \mathrm{GeV}\right]$ (see text), and assume the third slepton generation to be heavier by $60 \mathrm{GeV}$. Fig. 2.a is for $\operatorname{tg} \beta=2$ while fig. $2 . b$ is for $\operatorname{tg} \beta=40$.

Fig. 3: Contours of survival probability $P\left(\nu_{\mu} \rightarrow \nu_{\mu}\right)=0.8$ (solid lines) and 0.45 (dashed lines) for neutrino energies $E_{\nu}=10$ and $40 \mathrm{GeV}$, taking $\epsilon \equiv \Delta n_{\tau \mu} / \Delta n_{\mu e}=10^{-3}$.

Fig. 4: Differential $\nu_{\mu}$ (solid lines) and $\nu_{\tau}$ (dashed lines) yields $\left(\times z^{2}\right)$ vs. $z \equiv E_{\nu} / m_{\chi}$, for $\chi$ annihilations into $\tau$ pairs. Thin lines describe the original spectra (ref. [19]) while thick lines include the matter effects, assuming $m_{\chi}=50 \mathrm{GeV}$. The figures correspond to $\left(\epsilon, \Delta m^{2}, \sin ^{2} 2 \theta\right)$ equal to $\left(10^{-3}, 6 \times 10^{-4} \mathrm{eV}^{2}, 0.1\right)$ in fig. $4 . a,\left(10^{-3}, 3 \times 10^{-4} \mathrm{eV}^{2}, 0.1\right)$ in fig. $4 . b$ and $\left(10^{-3}, 10^{-3} \mathrm{eV}^{2}, 0.6\right)$ in fig. 4.c. 


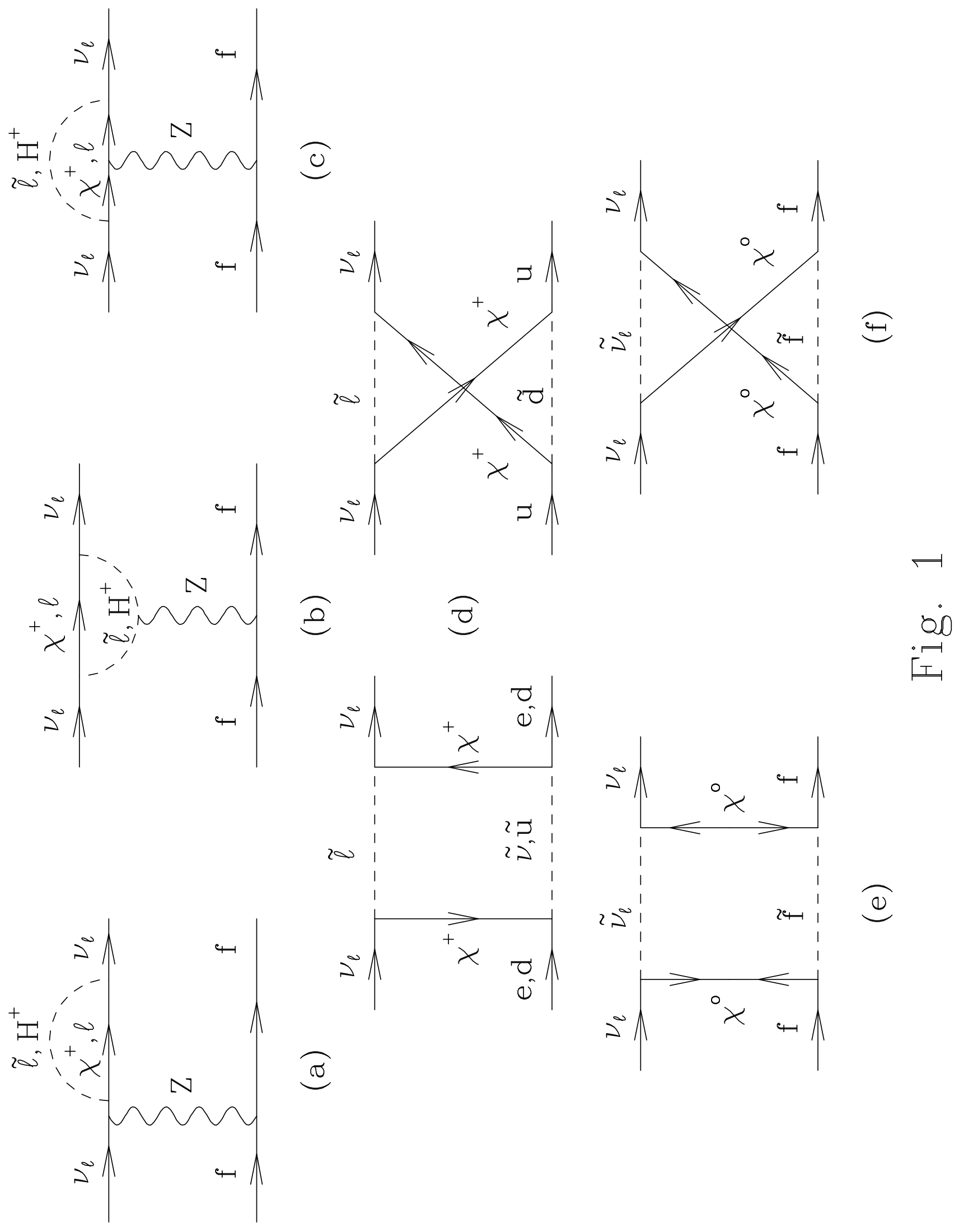




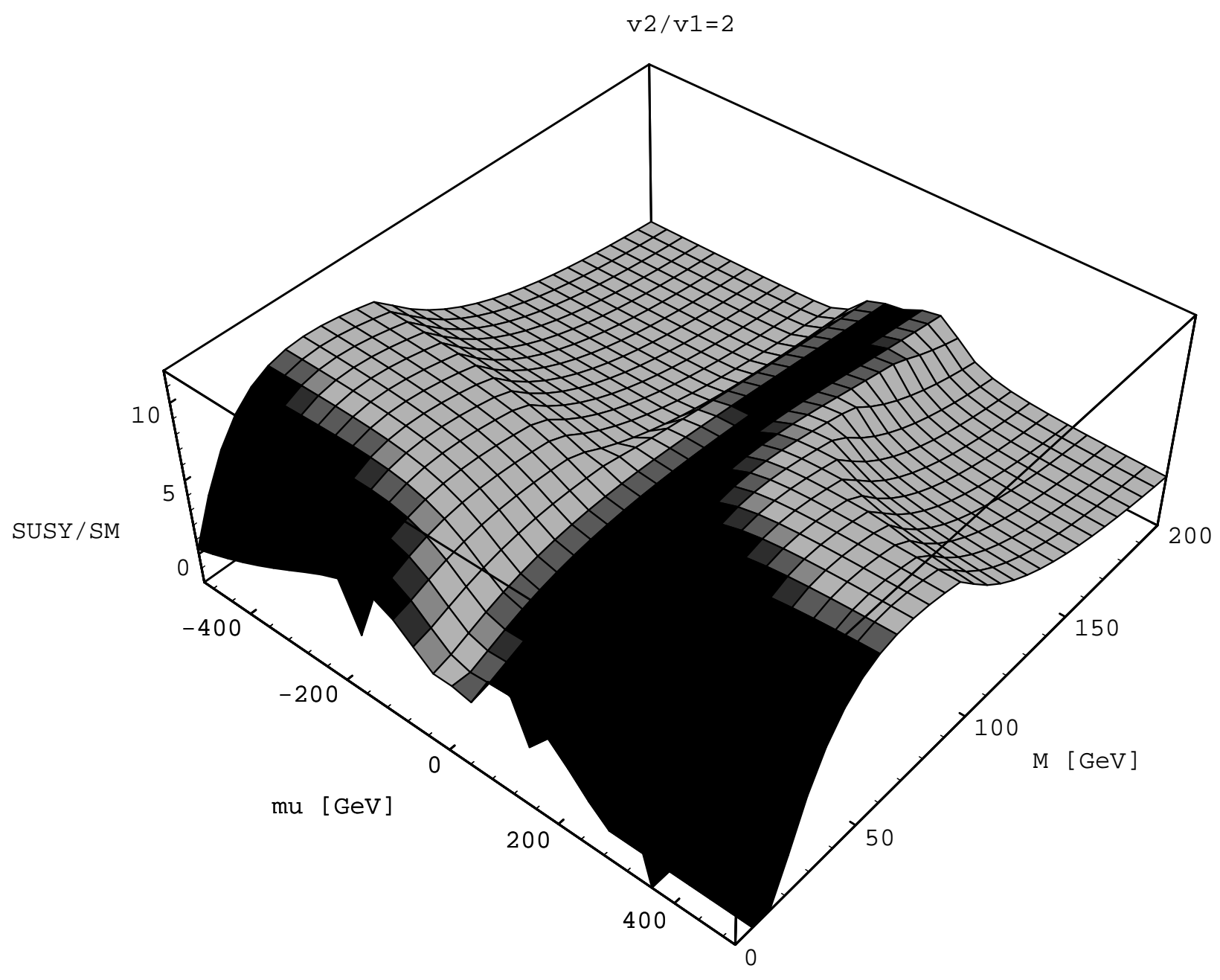




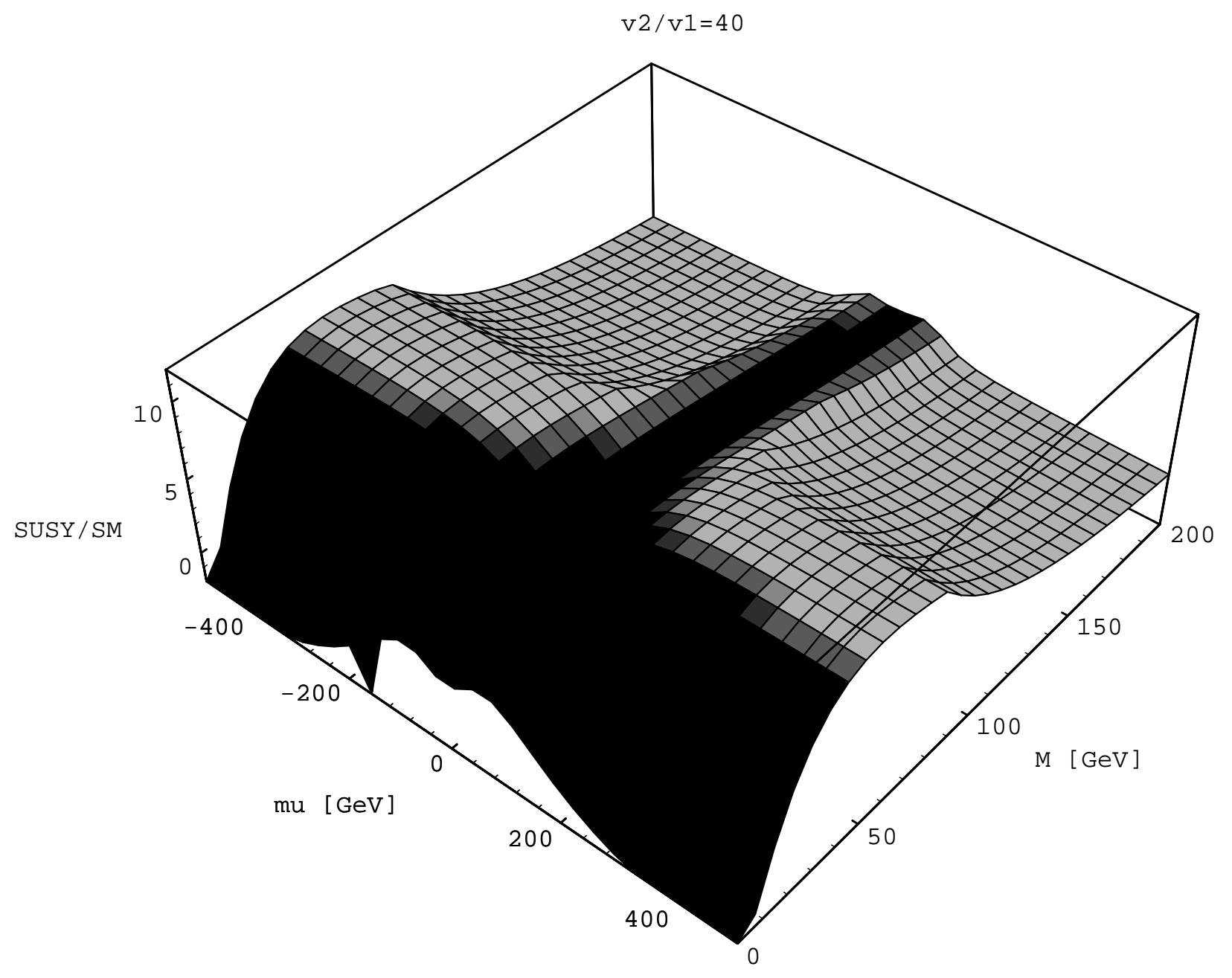




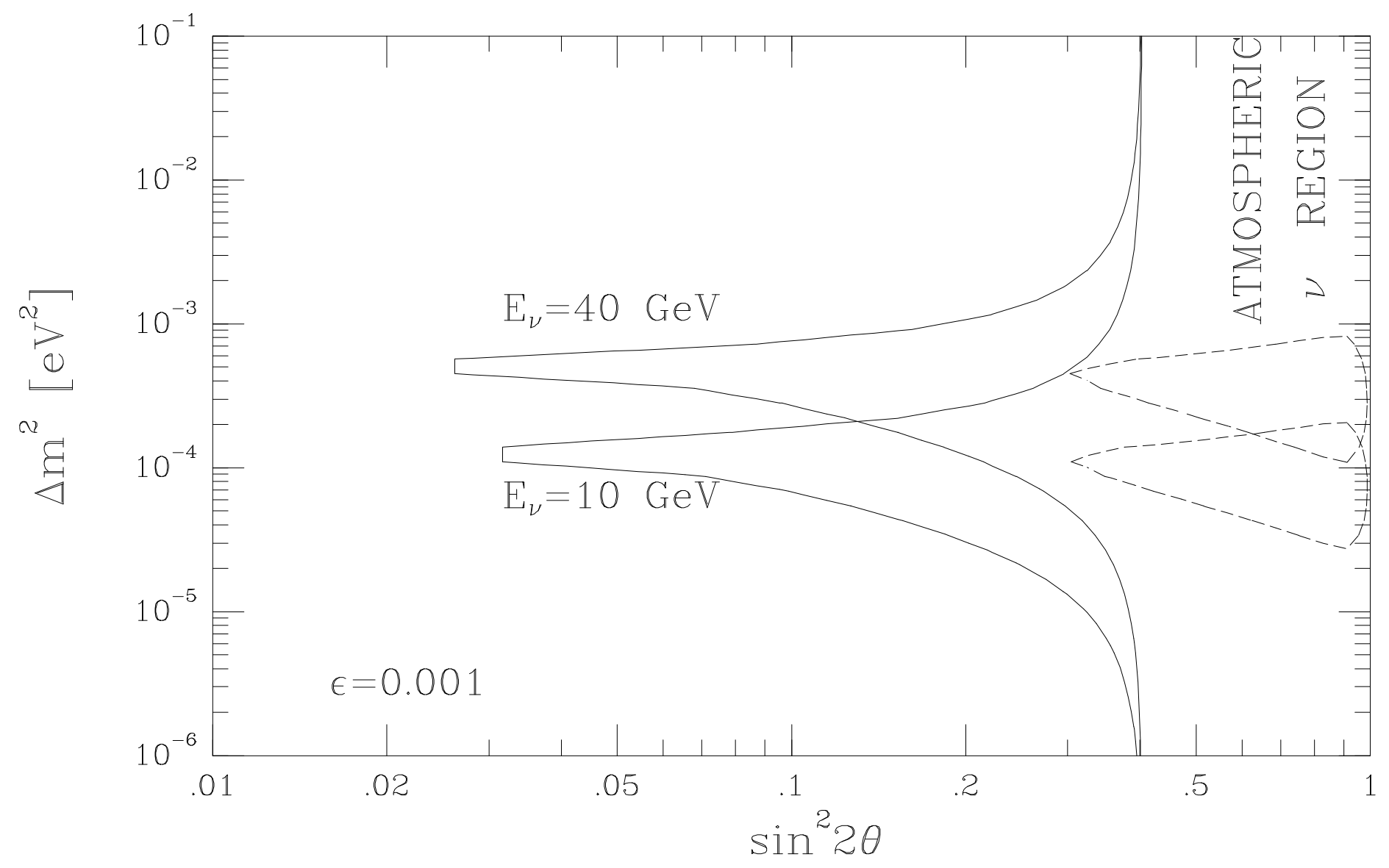




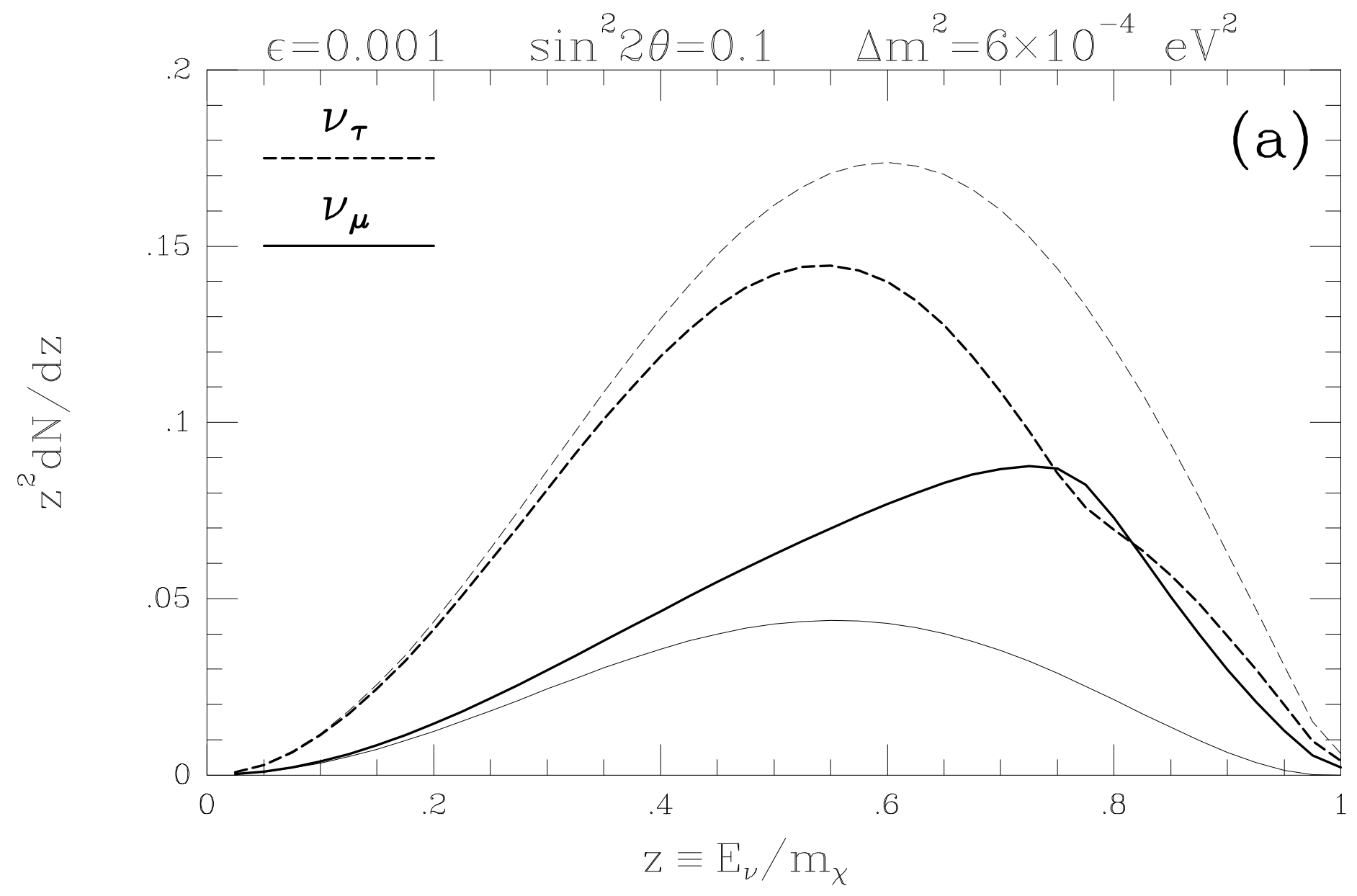




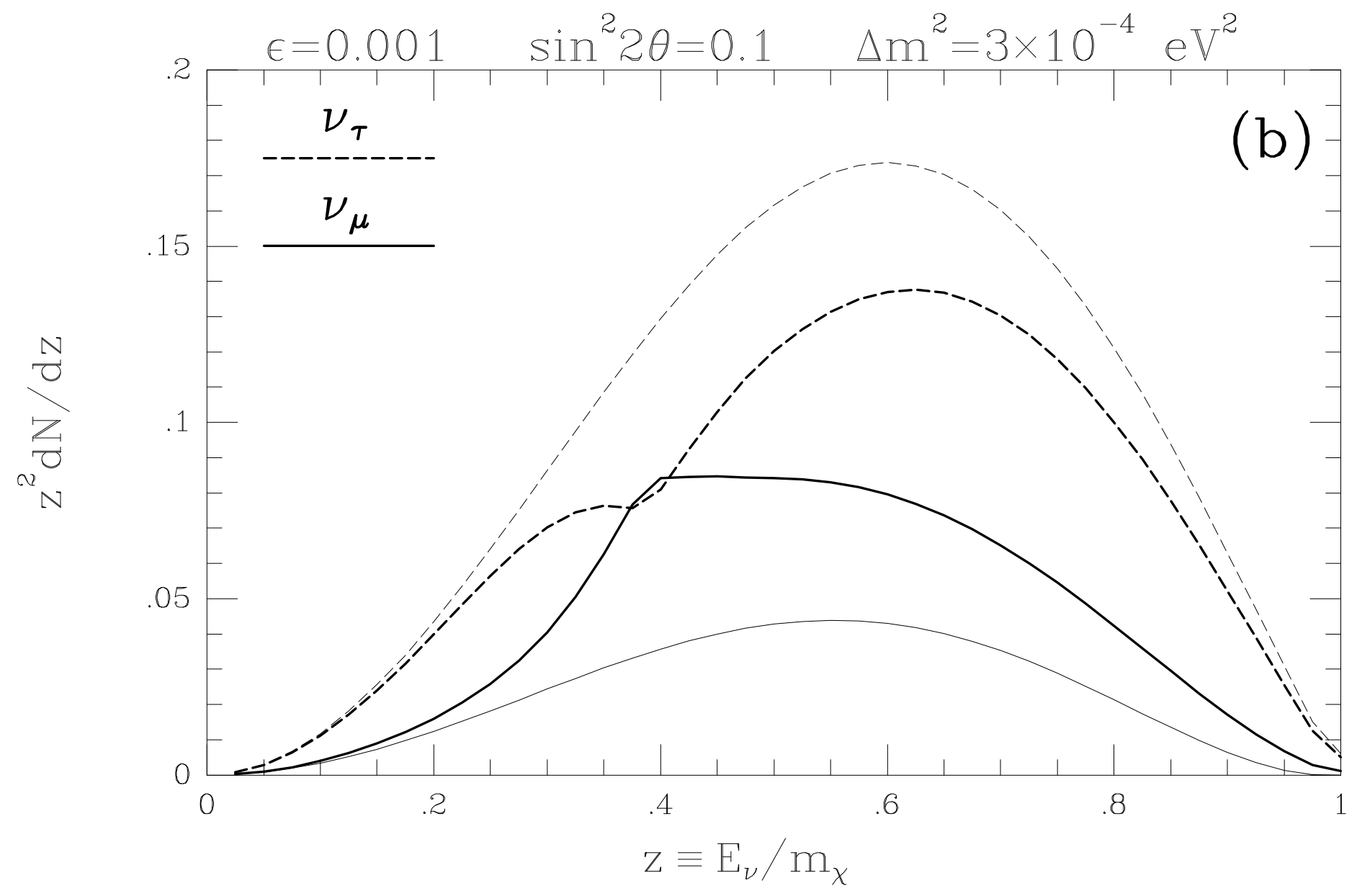




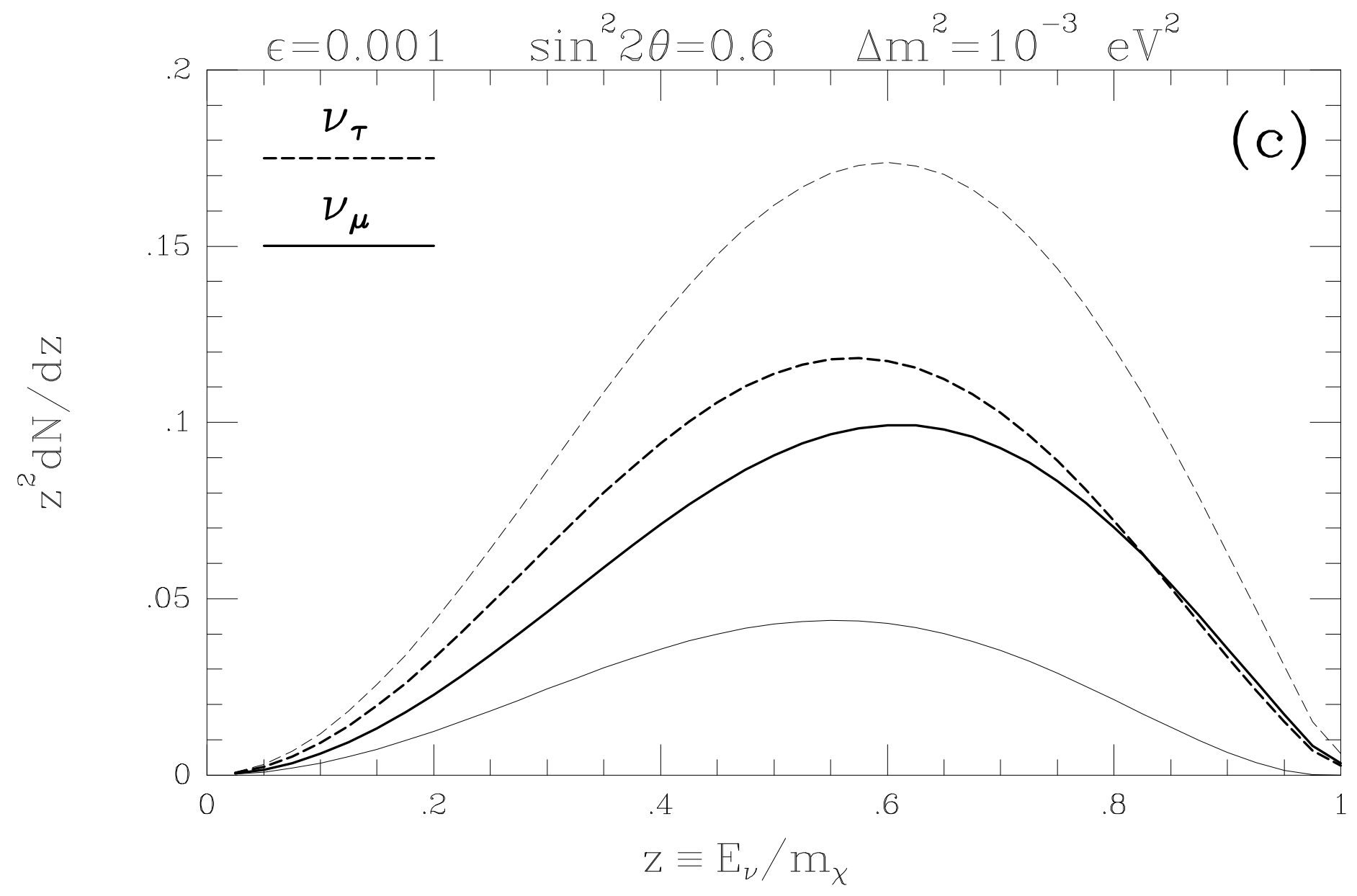

